\title{
Operant discrimination as a function of rate of stimulus alternation'
}

ALAN C. KAMIL AND JOHN W. DAVENPORT

REGIONAL PRIMATE RESEARCH CENTER, UNIVERSITY OF WISCONSIN

Three groups of rats were given operant discrimination training in which $S^{d} S^{\Delta}$ alternation occurred 3,6 , or 12 times per min. Only the $3 / \mathrm{min}$ group learned to inhibit $S^{\Delta}$ responses. The nonlearning of the $6 / \mathrm{min}$. and $12 / \mathrm{min}$. groups was discussed in terms of adventitious reinforcement of $S^{\Delta}$ responses

In the experimental procedure used to investigate differential conditioning in an operant situation, a number of stimuli, each signaling a particular schedule of reinforcement, are repeatedly presented to the organism. In the typical case, two stimuli are employed, one $\left(S^{d}\right)$ associated with reinforcement, the other $\left(S^{\Delta}\right)$ with nonreinforcement. When the alternation of the discriminative stimuli is programmed independently of the $S$ 's behavior, the schedule of $\mathrm{S}^{\mathrm{d}}-\mathrm{S}^{\Delta}$ alternation employed can vary along several temporally defined dimensions. The purpose of the present study was to investigate the effects of one temporal parameter, the frequency of $S^{d}-S^{\Delta}$ alternation, on the formation of an operant discrimination.

\section{Method}

The Ss were 12 experimentally naive male Holtzman rats, $120-130$ days old at the beginning of the experiment. They were maintained at $80 \%$ of their free-feeding weight for 14 days prior to, and throughout, the experiment. The apparatus consisted of two identical Gerbrands model $\mathrm{C}$ rat test chambers enclosed in picnic ice chests. The response panel in each was equipped with a retractable lever, two green one-inch cue lights, and a food cup. A small houselight was mounted above each chamber. A feeder which dispensed $45 \mathrm{mg}$ Noyes pellets, a small relay and a speaker were mounted behind each response panel. Throughout the experiment, $\mathrm{S}^{\mathrm{d}}$ was the click produced by $10 / \mathrm{sec}$. operation of the relay and $\mathbf{S}^{\Delta}$ was the $10 / \mathrm{sec}$. flashing of the cue lights. White noise was continuously presented through the speaker. The two chambers were controlled by standard programming equipment located in an adjacent room.

On the day before discrimination training began, each $\mathrm{S}$ was given 60 free pellets on a VI 1 schedule, with the bar retracted. The bar was then extended into the chamber and each $\mathrm{S}$ was allowed to make 40 reinforced responses. Neither $\mathrm{S}^{\mathrm{d}}$ nor $\mathrm{S}^{\Delta}$ was presented during this session. Discrimination training was run for 20 consecutive days. Each daily session lasted $16 \mathrm{~min}$. and was divided into $8 \mathrm{~min}$. each of $\mathrm{S}^{\mathrm{d}}$ and $\mathrm{S}^{\Delta}$. Every response during $S^{d}\left(R^{d}\right)$ and no response during $S^{\Lambda}\left(R^{\Lambda}\right)$ was reinforced. Each session began with onset of the houselight, extension of the lever into the chamber, and either an $S^{d}$ or $S^{\Delta}$ period. The stimulus which defined the first period was randomly determined, and the two discriminative stimuli simply alternated throughout the session. Three groups of four Ss, formed by random assignment, differed in the number of $S^{d}-S^{\Delta}$ alternations they received during training. Group 1 had 12 alternations/min. (5-sec. periods), group 2 had 6 alternations/ min. (10-sec. periods) and group 3 had 3 alternations/ min. (20-sec. periods). Three dependent variables were recorded for each session: number of $\mathrm{R}^{\Delta}$, number of $\mathrm{R}^{\mathrm{d}}$, and a derived discrimination index, $D I=R^{d} /\left(R^{d}+R^{\Delta}\right)$, the proportion of the total responses that were $R^{d_{S}}$. Results

As shown in the top panel of Fig. 1, only group 3 displayed any appreciable learning in terms of DI, reaching an apparent asymptote of about .80 , whereas
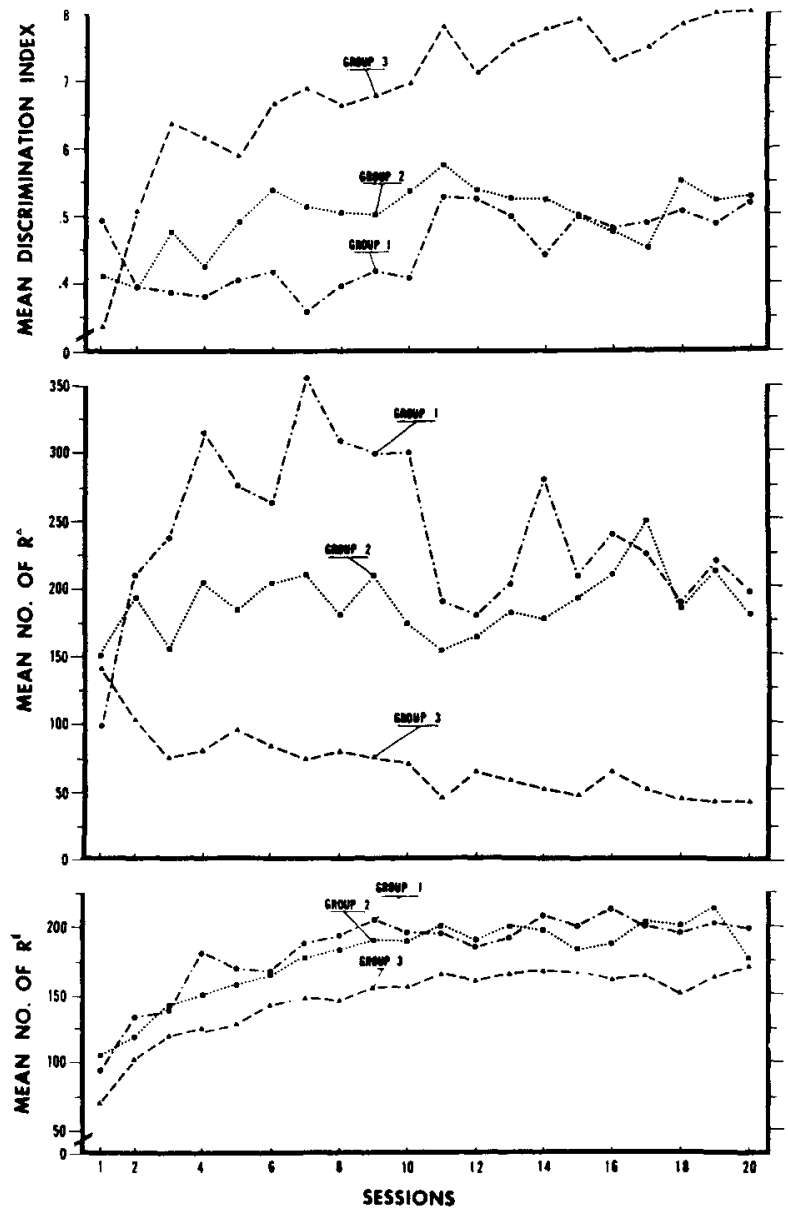

Fig. 1. Mean discrimination index (upper panel), mean number of responses during $\mathrm{s}^{\Lambda}$ (middle panel), and mean number of responses during $S^{d}$ (bottom panel) for the three groups. 
the other two groups were performing at about .50 after 20 days. A days-by-groups analysis of variance on these data yielded significant effects for groups $(p<.01)$, days $(p<.01)$ and the days-by-groups interaction $(p<.01)$. The middle panel of Fig. 1 , showing the number of $\mathbf{R}^{\Delta}$ across sessions, indicates that only group 3 showed any inhibition of $\mathrm{S}^{\Lambda}$ responding, while group 1 performed especially poorly on days 2-10. Analysis of variance of these data again yielded significant ground $(p<.01)$, days $(p<.05)$ and days-by-groups $(p<.05)$ effects. The bottom panel of Fig. 1, number of $R^{d}$ across sessions, shows all groups increasing their $\mathrm{S}^{\mathrm{d}}$ responding during training, with group 3 making fewer $R^{d_{s}}$ than the other groups. Analysis of variance of these data showed the groups $(p<.05)$ and the days $(p<.001)$ effects to be statistically reliable.

\section{Discussion}

The most interesting aspect of the results was the failure of groups 1 and 2 to learn to inhibit lever responses during $\mathrm{S}^{\Delta}$; these groups had $\mathbf{R}^{\Delta}$ asymptotes that were far above that of group 3, and group 1 showed a striking increase in $\mathrm{S}^{\Delta}$ responding over the first seven sessions, suggesting the action of a learning process. Among various possible interpretations of this high incidence of $\mathrm{S}^{\Lambda}$ responding, we find the most plausible to be in terms of adventitious reinforcement.
Assuming a random distribution of $R^{\Delta} \mathbf{s}$ across stimulus periods, Ss receiving a higher rate of stimulus alternation would be more likely to have a given $\mathbf{R}^{\Delta}$ followed by $\mathrm{S}^{\mathrm{d}}$ onset (itself a conditioned reinforcer after some training) and a reinforced $R^{d}$ than Ss having a low alternation rate. The reinforcement following $\mathrm{R}^{\mathrm{d}}$ would strengthen preceding $R^{\Delta}$ as well as $R^{d_{s}}$, tending to produce rapid chains of responding. In addition, viewing the independent variable in terms of length of a given stimulus period rather than the frequency of stimulus change, it seems likely that the differential probability of such adventitious reinforcement was increased by the greater opportunity for extinction to occur in the longer $\mathrm{S}^{\Delta}$ periods of group 3 and the possibility that frustration produces a temporarily heightened rate of lever responding very soon after $\mathrm{s}^{\Delta}$ onset.

This interpretation would suggest that the frequent stimulus alternation groups, particularly group 1, learned to emit chains of lever presses having short interresponse times during $\mathrm{S}^{\Delta}$. Such phenomenon would also explain the obtained $R^{\mathrm{d}}$ differences as due to a generalized tendency, in the groups that did not learn to inhibit $R^{\Delta_{S}}$, to respond in bursts of lever presses. Note

1. Supported by grant FR-00167 from the National Institutes of Health. 